\title{
Effects of Static, Stationary, and Traveling Trunk Exercises on Muscle Activation
}

\author{
Darien T. Pyka, Pablo B. Costa*, Jared W. Coburn, Lee E. Brown \\ Department of Kinesiology, California State University, Fullerton, 800 N. State College Blvd. USA
}

Corresponding Author: Pablo B. Costa, E-mail: pcosta@fullerton.edu

\section{ARTICLE INFO}

Article history

Received: August 11, 2017

Accepted: September 28, 2017

Published: October 31, 2017

Volume: 5 Issue: 4

Conflicts of interest: None

Funding: None

\begin{abstract}
Background: A new fitness trend incorporates stability exercises that challenges trunk muscles and introduces crawling as an exercise, but has yet to be investigated for muscle activity. Purpose: To compare the effects of static (STA), stationary (STN), and traveling (TRV) trunk exercises on muscle activation of the rectus abdominis, rectus femoris, external oblique, and erector spinae using surface electromyography (EMG). Methods: Seventeen recreationally active women (mean age $\pm \mathrm{SD}=22.4 \pm 2.4$ years, body mass $62.9 \pm 6.9 \mathrm{~kg}$, height $165.1 \pm 5.8 \mathrm{~cm})$ and twenty-three men $(23.6 \pm 3.9$ years, $83.2 \pm 17.1 \mathrm{~kg}, 177.1 \pm 9.1 \mathrm{~cm})$ volunteered to participate in this study. Subjects performed maximal voluntary contractions for normalization of each muscle's EMG activity. They then performed the three exercises in random order for thirty seconds each with a two-minute rest in between. Results: For the rectus abdominis, STA was significantly lower than $\operatorname{STN}(P=0.003)$ and TRV $(P=0.001)$. For the external oblique, STA was significantly lower than $\operatorname{STN}(P=0.001)$ and TRV $(P=0.001)$ and STN was significantly greater than TRV $(P=0.009)$. For the erector spinae and rectus femoris, STA was significantly lower than STN $(P=0.001)$ and TRV $(P=0.001)$ Conclusions: There was greater muscle activation in all muscles tested in the stationary and traveling exercises versus the static. Strength and conditioning coaches and allied health professionals could potentially use stationary and traveling forms of trunk stabilization exercises as a viable strategy to increase muscle activation.
\end{abstract}

Key words: Electromyography, Exercise Therapy, Torso, Muscle Contraction, Postural Balance, Back Pain

\section{INTRODUCTION}

The advancement in research and application of trunk exercises has benefited therapists, trainers, and coaches to improve sports performance, reduce injury risk, and in rehabilitation of their athletes or patients (McGill, 2010; Wheeler, 2015). Research has shown the most effective and safest method to train the trunk is a stabilization exercise, where a neutral spine is maintained against a load (Mendrin, Lynn, Griffith-Merritt, \& Noffal, 2016). This is due to increased knowledge that the most common function of the trunk is to prevent motion rather than initiate movement, and the trunk muscles should be trained as stabilizers rather than prime movers (McGill, 2010). McGill (2010) describes a stabilization exercise as any exercise that challenges the spine stability while enforcing trunk co-activation patterns that ensure a stable spine (McGill (2010). These exercises consist of holding the spine in a neutral position while the trunk is loaded through different strategies, such as moving upper and lower limbs in several positions or maintaining the pelvis lifted off the floor against gravity in a hold or stationary position (Vera-Garcia, Barbado, \& Moya, 2014). A neutral position is referred to as the natural curvature of the spine and the pelvis without an anterior or posterior tilt (McGill, 2010).

It is important to have a relatively strong trunk for resistance training and injury prevention (McGill, 2015). In a strongman study, McGill et al. (2009) concluded that strong trunk muscles allow force to dissipate distally to farther areas of the body. A stiff trunk allows power generated from the hips to be transmitted through the torso to the upper body or vice versa. It takes a stiff, stable trunk to allow optimal production, transfer, and control of force during a total body movement (Okada, Huxel, \& Nesser, 2011). Hodges and Richardson (1997) found the trunk stabilizers to be activated before any limb movements in a total body exercise, lending the support to the theory that movement control and stability is developed from the trunk to extremity (Okada et al., 2011). Many movements such as pushing, pulling, lifting, carrying, and rotation use power generated from the hips to perform the exercise (McGill, 2010). If a bend in the spine occurs, known as an "energy leak", power is compromised (McGill, 2010).

Chronic disabling low back pain prevalence is $4.2 \%$ in individuals between 24 and 39 years of age and 19.6\% in

Published by Australian International Academic Centre PTY.LTD.

Copyright (c) the author(s). This is an open access article under CC BY license (https://creativecommons.org/licenses/by/4.0/) http://dx.doi.org/10.7575/aiac.ijkss.v.5n.4p.26 
those between 20 and 59 years (Meucci, Fassa, \& Faria, 2015). There is a direct link between poor movement patterns and low back injuries. Common injuries are a result of excessive spinal flexion and trunk instability. In planning a trunk-strengthening program, stability and endurance exercises have been recommended to be first (McGill, 2010) and the most important (McGill, 2015). Improving the strength of the trunk without these two qualities increases the risk of not performing the exercise correctly with repetition and thereby increases the risk of injury (McGill, 2010). A training program focusing on neuromuscular control for trunk stabilization could be advantageous in a low back injury prevention program (Stevens et al., 2007; Wheeler et al., 2015). Such exercises include the four point kneeling bird dog with extension of the contra-lateral limbs and supine bridging with a single leg extension (Stevens et al., 2007).

Integration core exercises requiring activation of the distal trunk elicit greater activity of the primary abdominal and lumbar muscles compared to isolated exercises (Gottschall, Mills, \& Hastings, 2013). In addition, within a trunk stabilization exercise, as instability increases, trunk muscle activity increases proportionally (Anderson \& Behm, 2005). Common trunk stabilization exercises that incorporate the above techniques include variations of the prone bridge, side-bridge, quadruped bird-dog, and supine curl-up exercises (McGill, 2010). With the rising popularity of trunk stabilization training and the research on the importance for trunk stability and endurance, there is a new exercise trend known as movement flows that challenge the trunk in a traveling form that has yet to be tested for muscle activity. Part of this trend is the Animal Flow workout, coined by fitness trainer Mike Fitch, which is thought to specifically challenge trunk stability and endurance in a functional manner. In his Animal Flow Coaching Manual (2013) for his level one Animal Flow certification, the author describes the most common position of the Animal Flow workout to be the beast position or more commonly referred to as a bear crawl. This position mimics the bird dog by being a quadruped position but with the knees slightly raised off the ground. The bear position consists of a progression of a static hold, stationary contra-lateral arm and leg lift, and a traveling crawling movement, with the goal of maintaining a neutral spine in each. Accordingly, Mendrin et al. (2016) has already reported the bear crawl as an effective isometric trunk exercise.

In a quadruped position the exercise integrates distal muscle stabilizers and with the knees raised slightly off the ground it serves the same purpose as a plank, to maintain the pelvis position against gravity testing endurance. The exercise loads and challenges trunk coordination, neuromuscular coordination, and balance with the stationary contra-lateral limb lift and traveling crawling movement. This quadruped exercise appears to have the key concepts of a trunk stabilization exercise. However, every trunk exercise studied and listed is either a static hold or in a stationary position. Thus, the question remains of how traveling in a trunk exercise affects muscle activity. Therefore, the purpose of the present investigation was to compare muscle activation of the rectus abdominis, lumbar erector spinae, external oblique, and rectus femoris among static, stationary, and traveling exercises in the quadruped position using surface electromyography.

\section{METHODS}

\section{Subjects}

Seventeen recreationally active women (mean age $\pm \mathrm{SD}=22.4 \pm 2.4$ years, body mass $62.9 \pm 6.9 \mathrm{~kg}$, height $165.1 \pm 5.8 \mathrm{~cm})$ and twenty-three men $(23.6 \pm 3.9$ years, $83.2 \pm 17.1 \mathrm{~kg}, 177.1 \pm 9.1 \mathrm{~cm}$ ) volunteered to participate in this study. An a priori sample size estimate of 28 participants was determined using $G$ *Power software (version 3.1.9.2, Dusseldorf, Germany) with an effect size of 0.25 and a power level set at 0.8 . Participants met the inclusion criteria to participate in the study, which included being free of illness, injury, or physical disabilities that may inhibit optimal performance of the tested exercises. They were required to have a minimum of 3 years of recreational activity and have met ACSM's guidelines of 30 minutes of recreational activity at least 3 days a week for at least 3 months.

\section{Research Design}

All data were collected over two visits. The first session consisted of reading and signing an informed consent form, filling out a health status questionnaire, and familiarizing the participant with the equipment and exercises. This visit lasted approximately 30 minutes. Participants were instructed to shave areas needed for the surface electrodes before the second session. Areas included the back of their neck, right thigh, area around their navel, and low back. All participants were instructed to wear shorts, to not apply any lotion on their skin prior to the testing session, and women needed to wear a sports bra and remove any umbilicus piercings. The second session consisted of skinfold measurements (Lange Skinfold Caliper, Santa Cruz, CA, USA) on the electrode sites as well as areas for a three-site body composition analysis, electromyography (EMG) electrode attachment, maximal voluntary contractions, and testing of the three exercises for 30 seconds each in random order. Constant speed was set with a metronome at a speed of 55 beats per minute for the stationary and traveling exercise. Data collection for each participant took approximately 45 minutes.

In the first session, anthropometrics of each participant were measured, which consisted of their body mass and height. Body mass was measured and recorded in kilograms with a digital scale (Ohaus ES Series scale, Parsippany, NJ, USA). Height was measured with a stadiometer (Seca stadiometer, Chino, CA, USA) and recorded in centimeters. After, the participant practiced the correct form of the three exercises and became familiar with the cues the researcher would provide during testing. For the static exercise (STA), the participant was instructed to start in a quadruped position (Figure 1), with wrists placed directly under the shoulders, elbows straight, and knees directly under the navel with ankles dorsi-flexed. Once this position was correct, the participant was instructed to raise their knees slightly off the ground such that a neutral spine is maintained. Subjects 
rested once they performed this position correctly for 30 seconds. Throughout the practice and the start of each testing exercise, participants were given corrective instructions for proper technique. The researcher only provided corrective technique at the start of the testing exercises to assume the quadruped position and did not provide corrective cues during testing. Time was tracked via the EMG software.

After they rested, participants were instructed to get back into the correct static position. When the correct position was assumed, participants began practicing for the stationary (STN) by lifting their right hand off the ground by bending their elbow without losing their trunk position for two seconds. Once this was performed correctly, the participant practiced lifting their left foot off the ground by further flexing their knee without losing their trunk position for two seconds. Once this was performed correctly, they practiced raising their right hand and left foot in the technique they just practiced simultaneously without losing their trunk position for two seconds (Figure 2). The same protocol was followed for the left hand and right foot. When performed correctly, they practiced alternating contra-lateral limbs off the ground to the sound at the metronome. Once they performed this correctly for a total of 30 seconds, they rested for twenty seconds.

After they rested, they practiced the traveling exercise (TRV). Participants were instructed to get back into the correct static position. When the correct position was assumed, participants were instructed to raise their right hand, left foot off the ground simultaneously to the sound of the metronome, in the technique they performed earlier, and place them back down on the ground half a hands length from the stationary hand (Figure 3). They were instructed to raise their left hand, right foot with the same protocol to create a forward crawling movement. They crawled for approximately five to eight yards in the 30 seconds. Form was lost when trunk position or a neutral spine was not maintained or one wrist was not under the shoulder or one of their knees was not under the navel at all times.

\section{Maximum Voluntary Contraction}

In the second session, each participant performed a maximal voluntary isometric contraction of each measured muscle for normalization. Reproducing techniques from McGill and Karpowicz (2009), participants were instructed to push against the manual resistance provided by the research investigator for 10 seconds. For the abdominal muscles, participants assumed a sit-up position and were manually braced at the elbows. Participants then produced a maximal isometric flexor movement. With a 30 -second rest period, it was followed by a right twist movement for the external oblique. For the lumbar erector spinae, participants performed a resisted maximum extension in the Biering-Sorensen position. The participant lied prone on a plinth with the upper edge of the iliac crests aligned with the edge of the table (Demoulin, Vanderthommen, Duysens, \& Crielaard, 2006). A strap was placed around the knees to anchor the lower body to the table. With arms folded across the chest, participants were instructed to forcefully extend while being manually braced by the research investigator. For the rectus femoris, participants were positioned in a seated position and attempted an isometric knee extension with a simultaneous hip flexion. The same research investigator performed all the tests. The maximal amplitude in any normalizing contraction was used as the maximum for that muscle (McGill \& Karpowicz, 2009). After performing maximal voluntary contractions, the participant rested for two minutes. They then performed each exercise in random order for 30 seconds with a rest period of 2 minutes in between exercises to avoid fatigue. Exercises

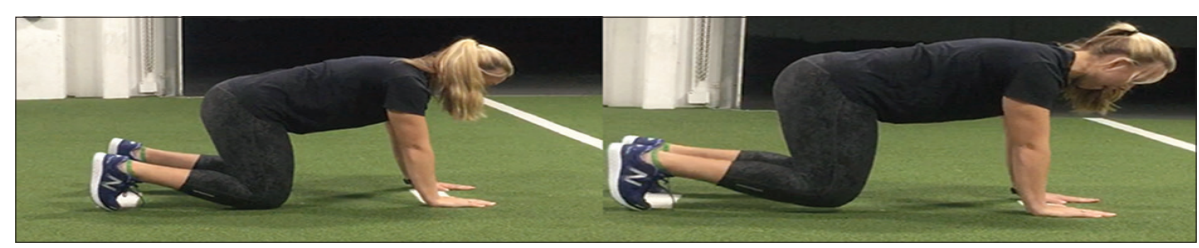

Figure 1. Static exercise (STA)

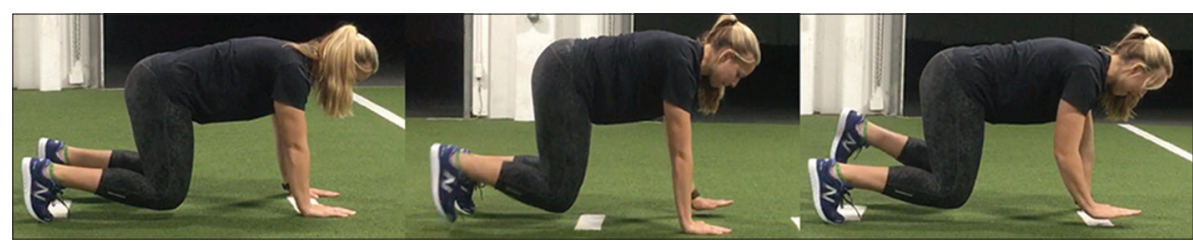

Figure 2. Stationary exercise (STN)

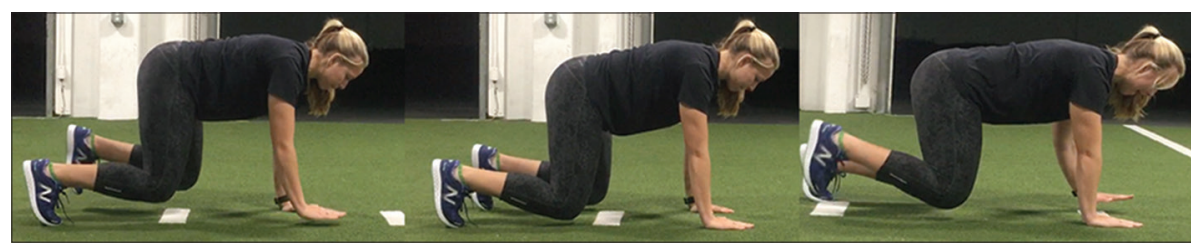

Figure 3. Traveling exercise (TRV) 
were performed for 30 seconds based on the recommendation by Mendrin et al. (2016).

All exercises were performed as practiced. If proper form was not maintained, the test was stopped and repeated until a full thirty seconds was performed correctly. Exercises were repeated if the correct form was not performed based on the investigator's (DTP) observations. Examples of incorrect form included not maintaining a neutral spine, raising hips into the air, dropping knees to the ground, or bending the elbows.

\section{Electromyography}

The participant's skin was prepared for the use of EMG electrodes prior to their placement, such that the signal was not distorted by exterior variables such as dead skin and excess hair. Excess hair at the site of the electrode placement was shaved and the site cleaned with a swab of isopropyl alcohol. Four preamplified, bipolar surface EMG electrodes (EL254S; Biopac Systems Inc., Santa Barbara, CA; gain $=350$ ) with a fixed center-to-center interelectrode distance of $20 \mathrm{~mm}$ were placed in accordance to the SENIAM guidelines (Hermans et al., 2000) and a previous investigation (McGill \& Karpowicz, 2009). Electrodes were placed unilaterally on the right side of body for the rectus femoris (RF), rectus abdominis (RA), external oblique (EO), and the lumbar erector spinae longissimus (ES). The RF electrode was placed midway on the line from the anterior superior iliac spine to the superior part of the patella. The RA electrode was placed $1 \mathrm{~cm}$ lateral to the navel. The EO electrode was placed $3 \mathrm{~cm}$ lateral to the linea semilunaris but on the same level of RA electrode. The ES electrode was placed $3 \mathrm{~cm}$ lateral from the spinous process of L1. A single pregelled, disposable electrode (EL501, Biopac Systems Inc., Santa Barbara, CA) was placed on the spinous process of the seventh cervical vertebrae to serve as a reference electrode.

The raw EMG signals were recorded simultaneously with a Biopac data acquisition system (MP150WSW; Biopac Systems Inc., Goleta, CA) interfaced with a laptop computer (Inspiron 8200; Dell Inc., Round Rock, TX) using proprietary software (AcqKnowledge version 5.0; Biopac Systems Inc.). Sampling frequency was set at $1000 \mathrm{~Hz}$ and the amplitude of the signals was expressed as root mean square (RMS) values. The EMG signals were bandpass filtered at $10-500 \mathrm{~Hz}$ and then normalized to their respective MVC. All analyses were performed with a custom program written with LabVIEW software (version 8.5, National Instruments, Austin, Texas). The middle 10-second epoch of the data collection from each exercise was used for analysis.

\section{Statistical Analysis}

Four separate two-way mixed factorial ANOVAs (exercise [static vs. stationary vs. traveling] $\times \operatorname{sex}[$ men vs. women]) were performed for each muscle. Post-hoc one- way ANOVAs were used when appropriate and necessary. An independent t- test was performed for body fat percentage between men and women. Four separate independent t-tests were performed for skinfold thickness of each site between men and women. An alpha of $P \leq 0.05$ was used to determine sta- tistical significance for all comparisons. Data were analyzed using SPSS version 23 software (SPSS Inc., Chicago, IL).

\section{RESULTS}

Table 1 contains means \pm SE for body fat percentage and skinfold thickness of the four electrode sites for men and women. There was a significant difference in body fat percentage between men and women $(P<0.001)$. In addition, there was a significant difference in skinfold thickness for the rectus femoris between men and women $(P=0.017)$. No significant differences in skinfold thickness were found between men and women for external oblique $(P=0.788)$, erector spinae $(P=0.884)$, or rectus abdominis $(P=0.864)$.

Table 2 contains means \pm SE for normalized EMG amplitude values for muscle activation under the three exercise conditions collapsed across sex. The results are separated by muscle and their differences in the static (STA), stationary (STN), and traveling (TRV) exercises. There was no significant interactions for sex $(P>.05)$; therefore, values are collapsed across sex.

For the rectus abdominis, there was no two-way interaction for exercise $\times \operatorname{sex}(P=0.789)$. However, there was a main effect for exercise $(P=0.002)$. Normalized EMG amplitude for STA was significantly lower than STN $(P=0.003)$ and TRV $(P=0.001)$ (Figure 4$)$. In addition, there was a main effect for sex $(P=0.001)$, where women had higher activation in all three exercises.

For the external oblique, there was no two-way interaction for exercise $\times \operatorname{sex}(P=0.287)$. However, there was a main effect for exercise $(P<0.001)$. Normalized EMG amplitude for STA was significantly lower than STN $(P=0.001)$ and TRV $(P=0.001)$ and STN was significantly greater than TRV $(P=0.009)$ (Figure 5). In addition, there was a main effect for $\operatorname{sex}(P=0.012)$ where women had higher activation in all three exercises.

For the erector spine, there was no two-way interaction for exercise $\times \operatorname{sex}(P=0.713)$. However, there was a main effect for exercise $(P<0.001)$. Normalized EMG amplitude for STA was significantly lower than $\operatorname{STN}(P=0.001)$ and TRV $(P=0.001)$ (Figure 6$)$. In addition, there was no main effect for $\operatorname{sex}(P=0.513)$.

For the rectus femoris, there was no two-way interaction for exercise $\times \operatorname{sex}(P=0.169)$. However, there was a main effect for exercise $(P<0.001)$. Normalized EMG amplitude

Table 1. Means \pm SE for body fat percentage and skinfold thickness

\begin{tabular}{lcc}
\hline Variables & Men & Women \\
\hline Body fat & $12.1 \pm 1.1^{*}$ & $21.3 \pm 0.8^{*}$ \\
RF & $15.7 \pm 1.5^{*}$ & $20.0 \pm 0.8^{*}$ \\
AB & $16.8 \pm 1.4$ & $17.2 \pm 1.5$ \\
EO & $14.7 \pm 1.6$ & $14.1 \pm 1.6$ \\
ES & $13.3 \pm 1.0$ & $13.1 \pm 0.8$ \\
\hline
\end{tabular}

$\mathrm{RF}=$ Rectus Femoris; $\mathrm{AB}=$ Rectus Abdominis; $\mathrm{EO}=$ External Oblique; ES=Erector Spinae, *significant difference between sexes 
Table 2. Normalized mean \pm SE for muscle activation under the three exercise conditions collapsed across sex

\begin{tabular}{lccc}
\hline Muscle & \multicolumn{3}{c}{ Exercise } \\
\cline { 2 - 4 } & STA & STN & TRV \\
\hline RF & $31.46 \% \pm 2.05 \%$ & $50.97 \% \pm 3.45 \% *$ & $52.07 \% \pm 3.67 \% *$ \\
AB & $18.31 \% \pm 2.51 \%$ & $26.52 \% \pm 3.43 \% *$ & $24.30 \% \pm 2.33 \% *$ \\
EO & $95.01 \% \pm 8.11 \%$ & $171.28 \% \pm 15.75 \% * *$ & $139.93 \% \pm 10.50 \% * *$ \\
ES & $6.24 \% \pm 0.60 \%$ & $13.18 \% \pm 1.20 \% *$ & $12.45 \% \pm 1.60 \% *$ \\
\hline
\end{tabular}

$\mathrm{STA}=$ Static; STN=Stationary; TRV=Traveling, $\mathrm{RF}=$ Rectus Femoris; $\mathrm{AB}=$ Rectus Abdominis; $\mathrm{EO}=$ External Oblique;

$\mathrm{ES}=$ Erector Spinae, *significant difference from STA exercise, ${ }^{*}$ significant difference among all exercises

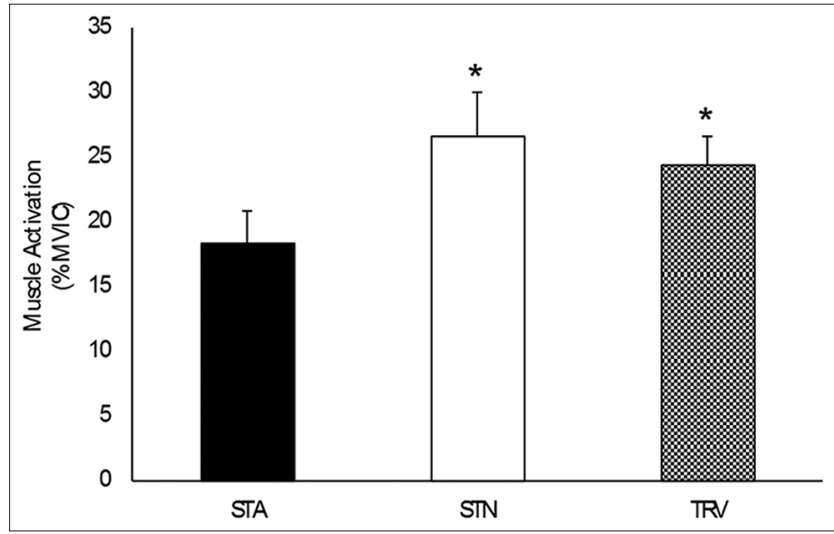

Figure 4. Mean $\pm \mathrm{SE}$ for normalized $\mathrm{EMG}$ amplitude of the rectus abdominis. STA = Static, STN = Stationary, TRV = Traveling,

*significant difference from STA

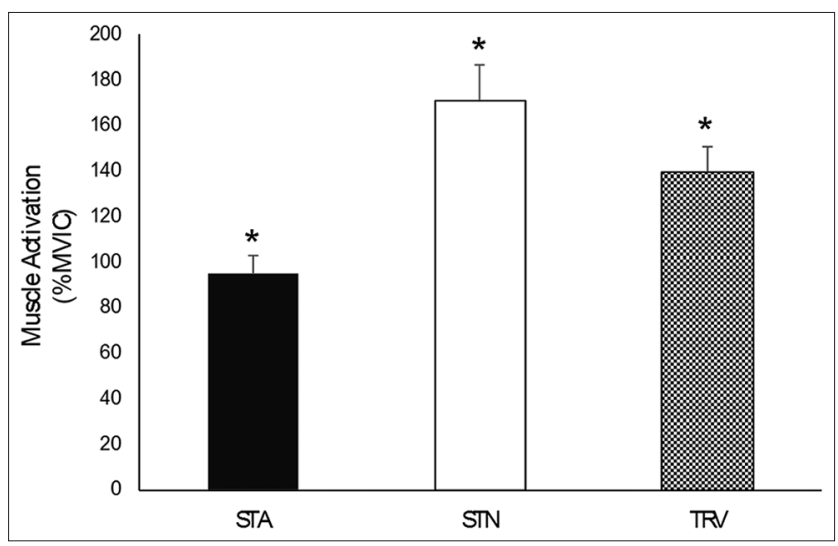

Figure 5. Mean \pm SE for normalized EMG amplitude of the external oblique. STA $=$ Static, STN $=$ Stationary, $\mathrm{TRV}=$ Traveling, *significant difference from STA

for STA was significantly lower than $\operatorname{STN}(P=0.001)$ and TRV $(P=0.001)$ (Figure 7$)$. In addition, there was no main effect for $\operatorname{sex}(P=0.886)$.

\section{DISCUSSION}

The results indicated there were differences in muscle activation among the static, stationary, and traveling trunk exercises. Based upon muscle activity, the exercises requiring movement of the upper and lower limbs elicited greater muscle activity while challenging coordination and balance. These findings are in congruence with Gottschall et

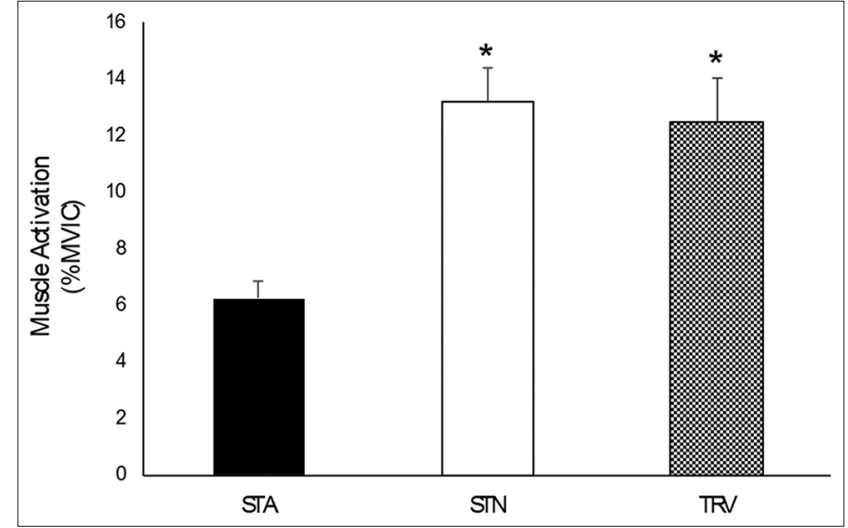

Figure 6. Mean \pm SE for normalized EMG amplitude of the erector spinae. $\mathrm{STA}=$ Static, $\mathrm{STN}=$ Stationary, $\mathrm{TRV}=$ Traveling, *significant difference from STA

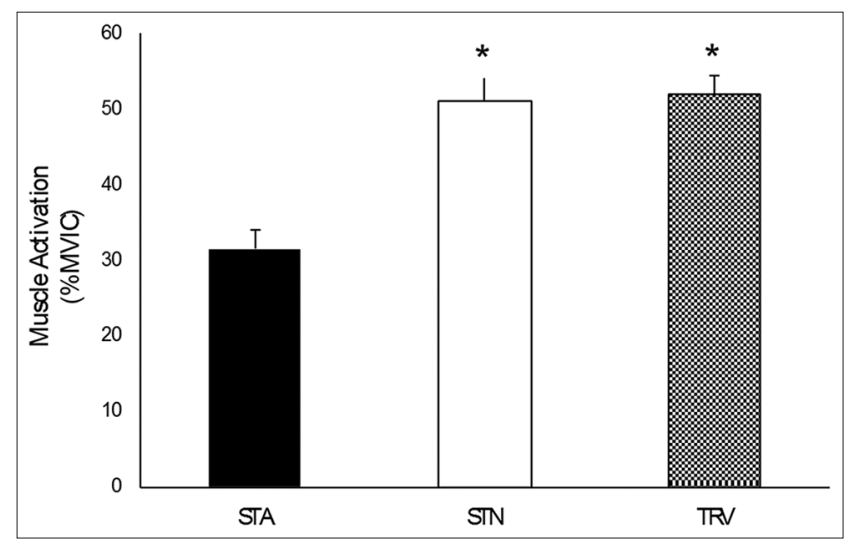

Figure 7. Mean $\pm \mathrm{SE}$ for normalized $\mathrm{EMG}$ amplitude of the rectus femoris. $\mathrm{STA}=$ Static, $\mathrm{STN}=$ Stationary, $\mathrm{TRV}=$ Traveling, *significant difference from STA

al. (2013) who reported integration core exercises requiring movement of the distal trunk, elicited higher activity of the primary abdominal and lumbar muscles compared to isolation exercises. The movement of the limbs with stabilization of the spine challenged postural stability and balance and resulted in greater muscle activation (Hanney, Pabian, Smith, $\&$ Patel, 2013). The current results are also in agreement with Anderson and Behm (2005) who reported as instability increases within a trunk stabilization exercise, trunk muscle activity increases. In addition, women had greater activation than men in all the exercises for the RA and EO. It is evident low back pain and injuries are common and on the rise 
(Hanney et al., 2013). A major contributing factor to this is trunk instability and lack of trunk muscle endurance (McGill, 2010). Exercises focusing on neuromuscular control for trunk stabilization could be advantageous in a low back injury prevention program (Stevens et al., 2007; Wheeler et al., 2015). Such exercises include the four-point kneeling bird dog with extension of the contra-lateral limbs, which contain similar elements as the stationary and traveling exercises tested in the present study.

The progression in muscle activation of static, stationary, and traveling exercises was shown to be correct for the rectus femoris. As for the rectus abdominis and erector spinae, the stationary exercise elicited greater muscle activation than the traveling exercise, albeit not significantly. Only the external oblique; however, showed a significant difference between the stationary and traveling exercise. Overall, the progression in movement from a static to stationary to traveling position did not show the same progression in level of muscle activity for the majority of the muscles tested. There was a main effect for sex for the rectus abdominis and the external oblique muscles. In all three exercises, women had greater relative muscle activation than men. This may be in part due to the exercise being relatively more difficult to perform for the women compared to the men and because of more strength-trained men participants than women. Therefore, the men may have had a stronger trunk and could utilize their strength more effectively in the exercises. Although in this case, greater muscle activation could not be explained by body composition, since women typically have higher levels of subcutaneous fat that could act as a filter. In the present study, women had significantly higher body fat percentage than men. Consequently, they had less fat-free mass and possibly needed greater activation to perform the exercises. Another possibility of women having greater muscle activation may be due to women adopting different motor recruitment strategies than men as reported in a study investigating unanticipated cutting maneuvers where women used different co-contraction strategies to achieve stabilization at the knee (Beaulieu, Lamontagne, \& Xu, 2008). However, in regards to muscle activity, no sex differences were found in a study comparing muscle activity in unilateral weight bearing tasks (Bouillon et al., 2012), trunk muscle activation during a squat and a deadlift compared to isometric instability exercises (Hamlyn, Behm, \& Young, 2007), and in various popular trunk exercises (Youdas et al., 2008).

McGill (2010) described that a strong and conditioned trunk musculature is needed to produce, control, and transfer force in various if not all movements. If the musculature cannot maintain strength to combat "energy leaks" or sustain a load over a long duration, the tissue will fatigue with each repetition and might eventually result in injury (McGill, 2010). Therefore, a compelling reason to strengthen the trunk musculature for adults or athletes is to decrease the chance of injury, especially low back and hip injuries as well as pain. Injury occurs when the applied load exceeds the strength of the tissue (McGill, 2015). More commonly, the injury results from the accumulation of repetitive micro-traumas when the tissue is fatigued (McGill, 2015). Strengthening the core through stabilization exercises improves postural control and the ability to land and decelerate the body, which increases the athlete's resistance to injury (Sadeghi, Shariat, Asadmanesh, \& Mosavat, 2013). Thus, it is important to incorporate exercises that challenge the strength and endurance of the trunk musculature in maintaining a neutral spine to prevent sub-traumas that will result in injuries. However, for improving sports performance, exercises targeting the trunk musculature might not be as beneficial and incorporating compound movements such as the front and back squats might be enough stress the trunk to improve strength and endurance (Tyler, Adams, \& DeBeliso, 2017). Nevertheless, for younger and less fit athletes, developing a proper foundation of trunk strength and endurance is essential to prevent future injury.

It has been researched and reported that the safest manner to train the trunk musculature is to maintain the spine in a neutral position when any load is placed on the body (Mendrin et al., 2016). Different strategies are used to place load on the body, including holding the pelvis off the floor and then moving the limbs in various positions (Vera-Garcia et al., 2014). The static, stationary, and traveling exercises used in this study fulfilled these loading properties. In addition, static exercises may be easier to learn and require less muscle activity. Therefore, static exercises are a good precursor for younger athletes and patients undergoing rehabilitation to acquire a foundation for trunk strength and endurance. Participants were limited to a population of convenience, which consisted of college-age participants from the local university and a strength and conditioning facility. In addition, no comparisons were made between individuals who were experienced in different modes of training (e.g., resistance, aerobic, etc.). Future studies include examining chronic effects of training with the exercises used in the present study and investigating participants already familiar with these exercises. Furthermore, future investigations may compare potential differences in balance and stability between static and stationary exercises training programs.

\section{CONCLUSION}

In conclusion, there was greater muscle activation in the stationary and traveling exercises compared to the static exercise. The next progression from a static exercise may be a stationary or traveling exercise. Other than the external oblique, there was no significance difference in muscle activity between stationary and traveling modes of trunk exercises. Due to the significant differences in muscle activity from the static mode, personal trainers, sports coaches, and allied health professionals who are seeking to increase instability during a trunk exercise, may wish to incorporate stationary and traveling variations into their training prescription. Stationary and traveling exercises with the movement of the limbs might potentially offer an alternative when training for trunk stabilization.

\section{REFERENCES}

Anderson, K., \& Behm, D. G. (2005). The impact of instability resistance training on balance and stability. Sports 
Medicine, 35(1), 43-53.

Beaulieu, M., Lamontagne, M., \& Xu, L. (2008). Gender differences in time-frequency EMG analysis of unanticipated cutting maneuvers. Medicine Science in Sports Exercise, 40(10), 1795- 1804.

Bouillon, L. E., Wilhelm, J., Eisel, P., Wiesner, J., Rachow, M., \& Hatteberg, L. (2012). Electromyographic assessment of muscle activity between genders during unilateral weight-bearing tasks using adjusted distances. International Journal of Sports Physical Therapy, 7(6), 595-605.

Demoulin, C., Vanderthommen, M., Duysens, C., \& Crielaard, J.-M. (2006). Spinal muscle evaluation using the Sorensen test: a critical appraisal of the literature. Joint Bone Spine, 73(1), 43-50.

Gottschall, J. S., Mills, J., \& Hastings, B. (2013). Integration core exercises elicit greater muscle activation than isolation exercises. The Journal of Strength \& Conditioning Research, 27(3), 590-596.

Hamlyn, N., Behm, D. G., \& Young, W. B. (2007). Trunk muscle activation during dynamic weight-training exercises and isometric instability activities. The Journal of Strength \& Conditioning Research, 21(4), 1108-1112.

Hanney, W. J., Pabian, P. S., Smith, M. T., \& Patel, C. K. (2013). Low back pain: movement considerations for exercise and training. Strength \& Conditioning Journal, 35(4), 99-106.

Hermens, H.J., Freriks, B., Disselhorst-Klug, C., Rau, G. Development of recommendations for SEMG sensors and sensor placement procedures. J Electromyogr Kinesiol 2000: 10: 361-374.

Hodges, P. W., \& Richardson, C. A. (1997). Contraction of the abdominal muscles associated with movement of the lower limb. Physical therapy, 77(2), 132-142.

McGill, S. M. (2010). Core training: evidence translating to better performance and injury prevention. Strength \& Conditioning Journal, 32(3), 33-46.

McGill, S. M. (2015). Low Back Disorders, 3E: Human Kinetics.

McGill, S. M., Grenier, S., Kavcic, N., \& Cholewicki, J. (2003). Coordination of muscle activity to assure stability of the lumbar spine. Journal of Electromyography and Kinesiology, 13(4), 353-359.

McGill, S. M., McDermott, A., Fenwick, C. M. (2009). Comparison of different strongman events: Trunk mus- cle activation and lumbar spine motion, load and stiffness. Journal of Strength \& Conditioning Research, 23(4), 1148-1161.

McGill, S. M., \& Karpowicz, A. (2009). Exercises for spine stabilization: motion/motor patterns, stability progressions, and clinical technique. Archives of Physical Medicine and Rehabilitation, 90(1), 118-126.

Mendrin, N., Lynn, S. K., Griffith-Merritt, H. K., \& Noffal, G. J. (2016). Progressions of isometric core training. Strength \& Conditioning Journal, 38(4), 50-65.

Meucci, R. D., Fassa, A. G., \& Faria, N. M. X. (2015). Prevalence of chronic low back pain: systematic review. Revista De Saude Publica, 49, 1-10.

Okada, T., Huxel, K. C., \& Nesser, T. W. (2011). Relationship between core stability, functional movement, and performance. The Journal of Strength \& Conditioning Research, 25(1), 252-261.

Sadeghi, H., Shariat, A., Asadmanesh, E., \& Mosavat, M. (2013). The effects of core stability exercise on the dynamic balance of volleyball players. International Journal of Applied Exercise Physiology, 2(2), 1-10.

Stevens, V. K., Coorevits, P. L., Bouche, K. G., Mahieu, N. N., Vanderstraeten, G. G., \& Danneels, L. A. (2007). The influence of specific training on trunk muscle recruitment patterns in healthy subjects during stabilization exercises. Manual Therapy, 12(3), 271-279.

Tyler, R., Adams, K. J., \& DeBeliso, M. (2017). The relationship between core stability \& squat ratio in resistance-trained males. International Journal of Kinesiology \& Sports Science, 5(2), 7-15.

Vera-Garcia, F. J., Barbado, D., \& Moya, M. (2014). Trunk stabilization exercises for healthy individuals. Revista Brasileira de Cineantropometria \& Desempenho Humano, 16(2), 200-211.

Wheeler, R. (2015). Limiting lower back injuries with proper technique and strengthening. Strength \& Conditioning Journal, 37(1), 18-23.

Youdas, J. W., Guck, B. R., Hebrink, R. C., Rugotzke, J. D., Madson, T. J., \& Hollman, J. H. (2008). An electromyographic analysis of the Ab-Slide exercise, abdominal crunch, supine double leg thrust, and side bridge in healthy young adults: implications for rehabilitation professionals. The Journal of Strength \& Conditioning Research, 22(6), 1939-1946. 